International Journal of Pure and Applied Mathematics

Volume $97 \quad$ No. $3 \quad 2014,359-367$

ISSN: 1311-8080 (printed version); ISSN: 1314-3395 (on-line version)

url: http://www.ijpam.eu

doi: http://dx.doi.org/10.12732/ijpam.v97i3.8

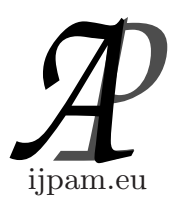

\title{
MATCHING AND EDGE COVERING NUMBER ON STRONG PRODUCT OF COMPLETE BIPARTITE GRAPHS
}

\author{
Thanin Sitthiwirattham \\ Department of Mathematics \\ Faculty of Applied Science \\ King Mongkut's University of Technology North Bangkok \\ Bangkok, 10800, THAILAND
}

\begin{abstract}
Let $\alpha^{\prime}(G)$ and $\beta^{\prime}(G)$ be the matching and edge covering number , respectively. The strong product $G_{1} \otimes G_{2}$ of graph of $G_{1}$ and $G_{2}$ has vertex set $V\left(G_{1} \otimes G_{2}\right)=V\left(G_{1}\right) \times V\left(G_{2}\right)$ and edge set $E\left(G_{1} \otimes G_{2}\right)=\left\{\left(u_{1}, v_{1}\right)\left(u_{2}, v_{2}\right) \mid\left[u_{1} u_{2} \in\right.\right.$ $E\left(G_{1}\right)$ and $\left.v_{1} v_{2} \in E\left(G_{2}\right)\right]$ or $\left[u_{1}=u_{2}\right.$ and $\left.v_{1} v_{2} \in E\left(G_{2}\right)\right]$ or $\left[u_{1} u_{2} \in E\left(G_{1}\right)\right.$ and $\left.\left.v_{1}=v_{2}\right]\right\}$. In this paper, we determined generalization of matching number and edge covering number on strong product of complete bipartite graphs and any simple graph.
\end{abstract}

AMS Subject Classification: 05C69, 05C70, 05C76

Key Words: strong product, edge covering number, matching number

\section{Introduction}

In this paper, graphs must be simple graphs. Let $G_{1}$ and $G_{2}$ be graphs. The strong product of graph $G_{1}$ and $G_{2}$, denote by $G_{1} \otimes G_{2}$, is the graph with $V\left(G_{1} \otimes G_{2}\right)=V\left(G_{1}\right) \times V\left(G_{2}\right)$ and $E\left(G_{1} \otimes G_{2}\right)=\left\{\left(u_{1}, v_{1}\right)\left(u_{2}, v_{2}\right) \mid\left[u_{1} u_{2} \in E\left(G_{1}\right)\right.\right.$ 
and $\left.v_{1} v_{2} \in E\left(G_{2}\right)\right]$ or $\left[u_{1}=u_{2}\right.$ and $\left.v_{1} v_{2} \in E\left(G_{2}\right)\right]$ or $\left[u_{1} u_{2} \in E\left(G_{1}\right)\right.$ and $\left.\left.v_{1}=v_{2}\right]\right\}$. There are some properties about strong product of graph. We recall these here.

Proposition 1. Let $H=G_{1} \otimes G_{2}=(V(H), E(H))$ then:

(i) $|V(H)|=\left|V\left(G_{1}\right)\right|\left|V\left(G_{2}\right)\right|$;

(ii) $|E(H)|=2\left|E\left(G_{1}\right)\right|\left|E\left(G_{2}\right)\right|+\left|V\left(G_{1}\right)\right|\left|E\left(G_{2}\right)\right|+\left|V\left(G_{2}\right)\right|\left|E\left(G_{1}\right)\right|$;

(iii) for every $(u, v) \in V(H), d_{H}((u, v))=d_{G_{1}}(u) d_{G_{2}}(v)+d_{G_{1}}(u)+d_{G_{2}}(v)$.

Theorem 2. Let $G_{1}$ and $G_{2}$ be connected $g$ graphs, The graph $H=$ $G_{1} \otimes G_{2}$ is connected if and only if $G_{1}$ or $G_{2}$ contains an odd cycle.

Next we get that general form of graph of strong product of $K_{m, n}$ and a simple graph.

Proposition 3. Let $G$ be a connected graph with order $p, V(G)=\left\{v_{j} / j=\right.$ $1,2, \ldots, p\}$ and $V\left(K_{m, n}\right)=\left\{u_{i} / i=1,2, \ldots, m+n\right\}$, the graph of

$$
K_{m, n} \otimes G \cong \bigcup_{i=1}^{m} H_{i} \cup \bigcup_{i=1}^{m+n} R_{i} \cup \bigcup_{j=1}^{p} S_{j} ; H_{i}=\bigcup_{j=m+1}^{m+n} H_{i j},
$$

where

$$
V\left(H_{i j}\right)=W_{i} \cup W_{j}, \quad W_{i}=\left\{\left(u_{i}, v_{1}\right),\left(u_{i}, v_{2}\right), \ldots,\left(u_{i}, v_{p}\right)\right\}, \quad i<j
$$

and

$$
E\left(H_{i j}\right)=\left\{\left(u_{i}, v\right)\left(u_{j}, w\right) / v w \in E(G)\right\},
$$

$V\left(R_{i}\right)=W_{i}$ and $E\left(R_{i}\right)=\left\{\left(u_{i}, v\right)\left(u_{i}, w\right) / v w \in E(G)\right\}$,

$$
V\left(S_{j}\right)=\left\{\left(u_{1}, v_{j}\right),\left(u_{2}, v_{j}\right), \ldots,\left(u_{m+n}, v_{j}\right)\right\}
$$

and

$$
E\left(S_{j}\right)=\left\{\left(u, v_{j}\right)\left(w, v_{j}\right) / u w \in E\left(K_{m, n}\right)\right\} .
$$

Moreover, if $G$ has no odd cycle then each $H_{i j}$ has exactly two connected components isomorphic to $G$.

Example

Next, we give the definitions about some graph parameters. A subset of the edge set $E$ of $G$ is said to be matching or an independent edge set of $G$, if no two distinct edges in $M$ have a common vertex. A matching $M$ is maximum matching in $G$ if there is no matching $M^{\prime}$ of $G$ with $\left|M^{\prime}\right|>|M|$. The cardinality 


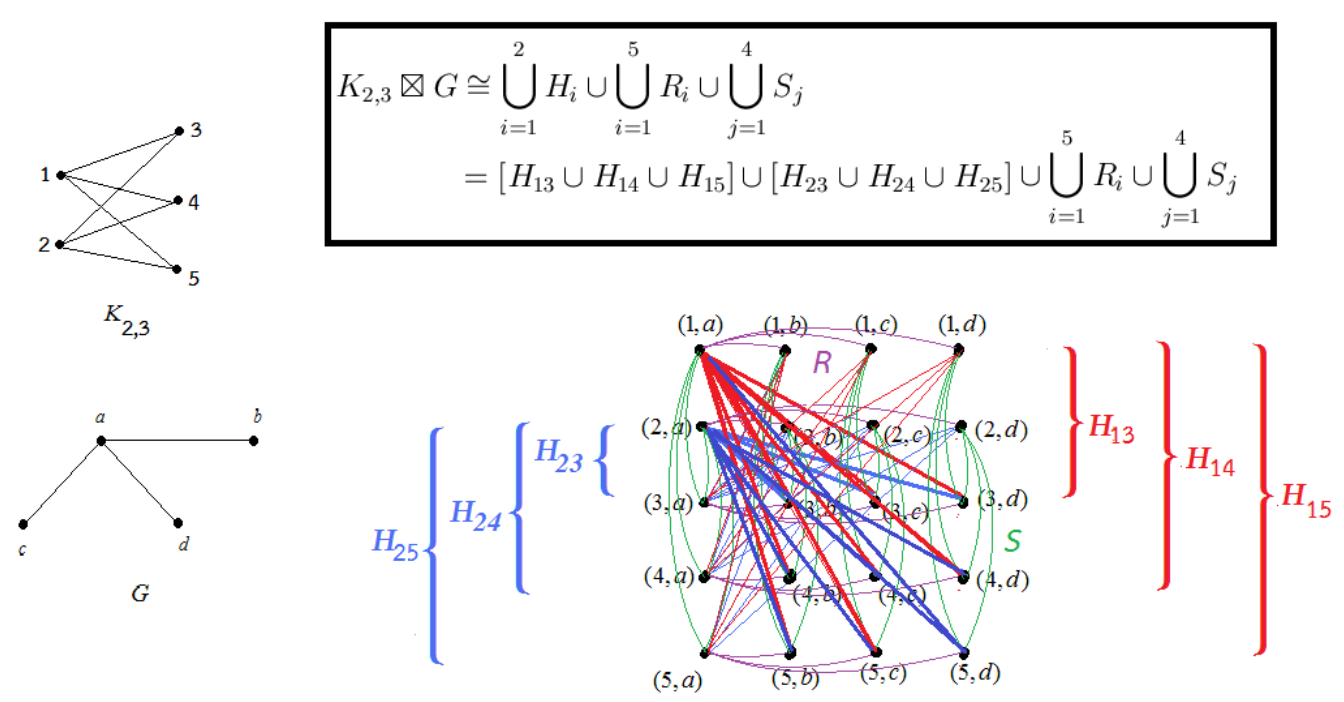

Figure 1: The graph of $K_{2,3} \otimes G$

of maximum matching of $G$ is called the matching number of $G$, denoted by $\alpha^{\prime}(G)$.

An edge of graph $G$ is said to cover the two vertices incident with it, and an edge cover of a graph $G$ is a set of edges covering all the vertices of $G$. The minimum cardinality of an edge cover of a graph $G$ is called the edge covering number of $G$, denoted by $\beta^{\prime}(G)$.

By definitions of edge covering number and matching number, clearly that $\alpha^{\prime}\left(K_{m, n}\right)=\min \{m, n\}$ and $\beta^{\prime}\left(K_{m, n}\right)=\max \{m, n\}$.

\section{Matching Number of the Graph of $K_{m, n} \otimes G$}

We begin this section by giving the lemma 4 show character of maximum matching for each $H_{i j}, R_{i}$ and $S_{j}$.

Lemma 4. Let $K_{m, n} \otimes G \cong \bigcup_{i=1}^{m} H_{i} \cup \bigcup_{i=1}^{m+n} R_{i} \cup \bigcup_{j=1}^{p} S_{j} ; \quad H_{i}=\bigcup_{j=m+1}^{m+n} H_{i j}$. Then $\alpha^{\prime}\left(H_{i j}\right)=2 \alpha^{\prime}\left(R_{i}\right)=2 \alpha^{\prime}(G)$ and $\alpha^{\prime}\left(S_{j}\right)=\min \{m, n\}$.

Proof. Suppose $G$ has no odd cycle, by proposition 3, we have that $H_{i j}$ contains 2 components isomorphic to $G$. Therefore $H_{i j}=2 G$. So $\alpha^{\prime}\left(H_{i j}\right)=$ 
$2 \alpha^{\prime}(G)$.

If $\mathrm{G}$ has odd cycle, then we have $\left.\left.d_{H_{\mathrm{ij}}}\left(\left(u_{i}, v\right)\right)=d_{H_{\mathrm{ij}}}\left(u_{j}, v\right)\right)=d_{G}(v)\right)$ for $\left(u_{i}, v\right) \in W_{i}$ and $\left(u_{j}, v\right) \in W_{j}, \quad i<j$.

In the rest of the proof we will consider $H_{i j}$. We first note that by definition of tensor product of $H_{i j}$, that for all $v, x \in G,\left(u_{i}, v\right)$ and $\left(u_{i}, x\right)$ are not joined by an edge, and that because the vertices $u_{i}, u_{j}$ are always joined by an edge, two vertices $\left(u_{i}, v\right),\left(u_{j}, w\right) \in H_{i j}$ are joined by an edge if and only if the two vertices are joined by an edge.

We now prove that $\alpha^{\prime}\left(H_{i j}\right)=2 \alpha^{\prime}(G)$.

(1) We first prove that if two vertices $v, w$ are matched in $G$, then the two vertices $\left(u_{i}, v\right),\left(u_{j}, w\right)$ can also be matched in $H_{i j}$ and the two vertices $\left(u_{i}, w\right),\left(u_{j}, v\right)$ can also be matched in $H_{i j}$. As stated above, for all vertices $v, w \in G$, edges $\left(u_{i}, v\right),\left(u_{i}, w\right)$ and $\left(u_{j}, v\right),\left(u_{j}, w\right)$ cannot exist. However, if $v, w$ are matched vertices in $G$, then the pair $\left(u_{i}, v\right),\left(u_{j}, w\right)$ can be matched in $H_{i j}$ and the pair $\left(u_{j}, v\right),\left(u_{i}, w\right)$ can also be matched in $H_{i j}$ because edge $v w$ exists in $G$. Therefore, the result follows.

(2) We next prove that if $v, w \in G$ cannot be matched in $G$, then the pair $\left(u_{i}, v\right),\left(u_{j}, w\right)$ cannot be matched in $H_{i j}$ and the pair $\left(u_{j}, v\right),\left(u_{i}, w\right)$ cannot be matched in $H_{i j}$. The reason is that $v, w \in G$ cannot be matched only if the edge $v w$ does not exist and therefore an edge does not exist between vertices $\left(u_{i}, v\right),\left(u_{j}, w\right) \in H_{i j}$ and between vertices $\left(u_{j}, v\right),\left(u_{i}, w\right) \in H_{i j}$. Therefore two vertices matched in $\mathrm{G}$ correspond to two possible matchings in and if two vertices cannot be matched in $\mathrm{G}$ then no vertices in the tensor product containing these two vertices can be matched.

Hence a matched set in $G$ will correspond to two matched sets in $H_{i j}$ and therefore $\alpha^{\prime}\left(H_{i j}\right)=2 \alpha^{\prime}\left(R_{i}\right)=2 \alpha^{\prime}(G)$ [since $\left.R_{i} \cong G\right]$. From $S_{j} \cong K_{m, n}$, we get $\alpha^{\prime}\left(S_{j}\right)=\alpha^{\prime}\left(K_{m, n}\right)=\min \{m, n\}$.

Definition 5. Given a matching $M$, an $M$-alternating path is a path that alternates between edges in $M$ and edges not in $M$. An $M$-alternating path whose endpoints are unsaturated by $M$ is an $M$-augmenting path.

Theorem 6. A matching $M$ in a graph $G$ is a maximum matching in $G$ if and only if $G$ has no $M$-augmenting path.

Next, we establish theorem 5 for a matching number of $K_{m, n} \otimes G$.

Theorem 7. Let $G$ be a connected graph with order $p$, then

$$
\alpha^{\prime}\left(K_{m, n} \otimes G\right)= \begin{cases}p m, & m=n \\ p n+(m-n) \alpha^{\prime}(G), & m>n \\ p m+(n-m) \alpha^{\prime}(G), & m<n\end{cases}
$$


Proof. Let $V\left(K_{m, n}\right)=\left\{u_{i} / i=1,2, \ldots, m+n\right\}$ and $V(G)=\left\{v_{j} / j=\right.$ $1,2, \ldots, p\}$. Since $\alpha^{\prime}\left(K_{m, n}\right)=\min \{m, n\}$. Let $\alpha^{\prime}(G)=k$, assume that the maximum matching of $K_{m, n}, G$ be

$$
\begin{gathered}
M_{1}=\left\{\begin{array}{l}
\left\{u_{1} u_{m+1}, u_{2} u_{m+2}, \ldots, u_{m} u_{2 m}\right\} \\
\left\{u_{1} u_{m+1}, u_{2} u_{m+2}, \ldots, u_{n} u_{m+n}\right\}
\end{array} \quad \text { where } m \leq n\right. \\
M_{2}=\left\{v_{j} v_{j+1} / j=1,3, \ldots, 2 k-1\right\} \text { respectively. }
\end{gathered}
$$

Case 1. $m=n$. We have $M_{1}$ is a maximum matching in $K_{m, n}$. Let $S_{j}^{*}=\left\{\left(u, v_{j}\right)\left(w, v_{j}\right) \mid u w \in M_{1}\right\} ; j=1,2, \ldots, p$. Therefore a maximum matching in $K_{m, n} \otimes G$ is $\bigcup_{j=1}^{p} S_{j}^{*}$.

Hence $\alpha^{\prime}\left(K_{m, n} \otimes G\right)=\left|\bigcup_{j=1}^{p} S_{j}^{*}\right|=p m$ where $m=n$.

Case 2. $m>n$. We have the matching $\bigcup_{j=1}^{p} S_{j}^{*}$. But $\bigcup_{i=1}^{n-1} R_{m+i}$ is not matched yet. Let $R_{m+i}^{*}=\left\{\left(u_{m+i}, v\right)\left(u_{m+i}, w\right) \mid v w \in M_{2}\right\} ; i=1,2, \ldots, n-1$. So we get $\bigcup_{j=1}^{p} S_{j}^{*} \cup \bigcup_{i=1}^{n-1} R_{m+i}^{*}$ is matching in $\left.K_{m, n} \otimes G\right)$.

So $\alpha^{\prime}\left(K_{m, n} \otimes G\right) \geq\left|\bigcup_{j=1}^{p} S_{j}^{*}\right|+\left|\bigcup_{i=1}^{n-1} R_{m+i}^{*}\right|=p n+(m-n) \alpha^{\prime}(G)$.

Suppose that $\alpha^{\prime}\left(K_{m, n} \otimes G\right)>p n+(m-n) \alpha^{\prime}(G)$, then there exist an augmenting path for $\bigcup_{j=1}^{p} S_{j}^{*} \cup \bigcup_{i=1}^{n-1} R_{m+i}^{*}$. That is not true because each edges in $K_{m, n} \otimes G$ incident with edge in $\bigcup_{j=1}^{p} S_{j}^{*} \cup \bigcup_{i=1}^{n-1} R_{m+i}^{*}$.

Hence $\alpha^{\prime}\left(K_{m, n} \otimes G\right)=p n+(m-n) \alpha^{\prime}(G)$ where $m>n$.

Case 3. $m<n$. In the same as case 2, we have $\alpha^{\prime}\left(K_{m, n} \otimes G\right)=\left|\bigcup_{j=1}^{p} S_{j}^{*}\right|+$ $\left|\bigcup_{i=1}^{n-m} R_{2 m+i}^{*}\right|=p m+(n-m) \alpha^{\prime}(G)$ where $m<n$. 


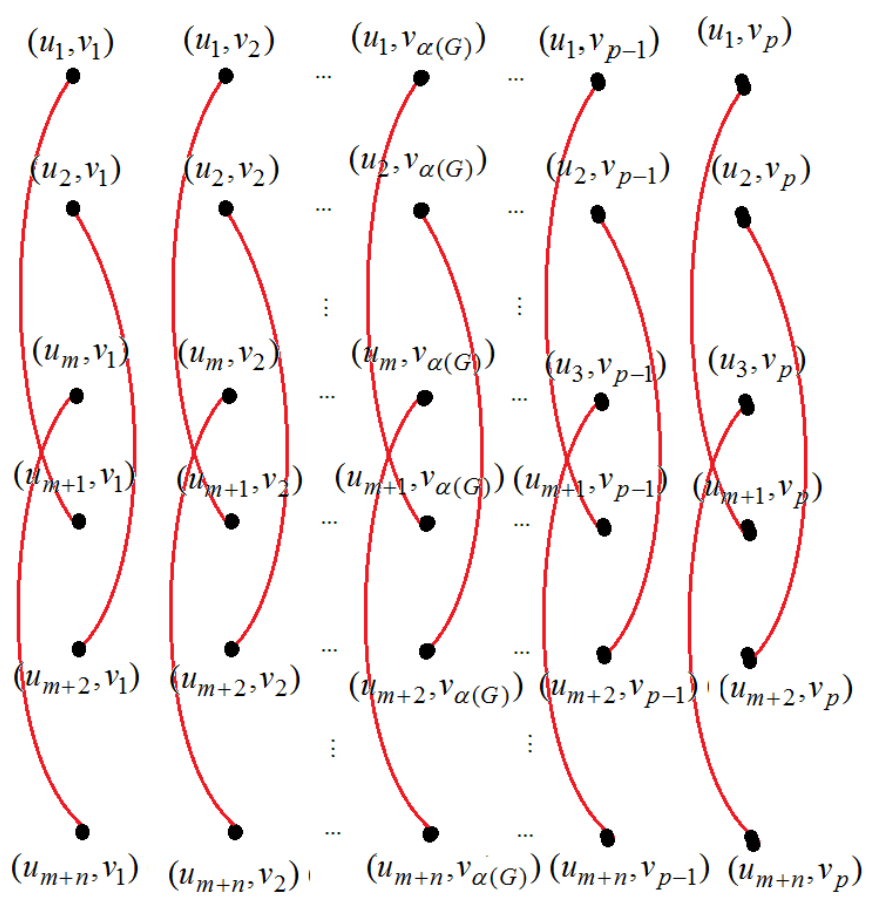

Figure 2: The case of $m=n$

\section{Edge Covering Number of the Graph of $K_{m, n} \otimes G$}

We begin this section by by giving the lemma 8 that shows a relation of matching number and edge covering number.

Lemma 8. Let $G$ be a simple graph with order $n$. Then $\beta^{\prime}(G)+\alpha^{\prime}(G)=n$

Next we establish theorem 9 for a edge covering number of $K_{m, n} \otimes G$.

Theorem 9. Let $G$ be a connected graph with order $p$, then

$$
\beta^{\prime}\left(K_{m, n} \otimes G\right)= \begin{cases}p m, & m=n \\ p n+(m-n) \beta^{\prime}(G), & m>n \\ p m+(n-m) \beta^{\prime}(G), & m<n\end{cases}
$$

Proof. By theorem 7 and lemma 8, we can also show that $\alpha^{\prime}\left(K_{m, n} \otimes G\right)+$ $\beta^{\prime}\left(K_{m, n} \otimes G\right)=(m+n) p$. 


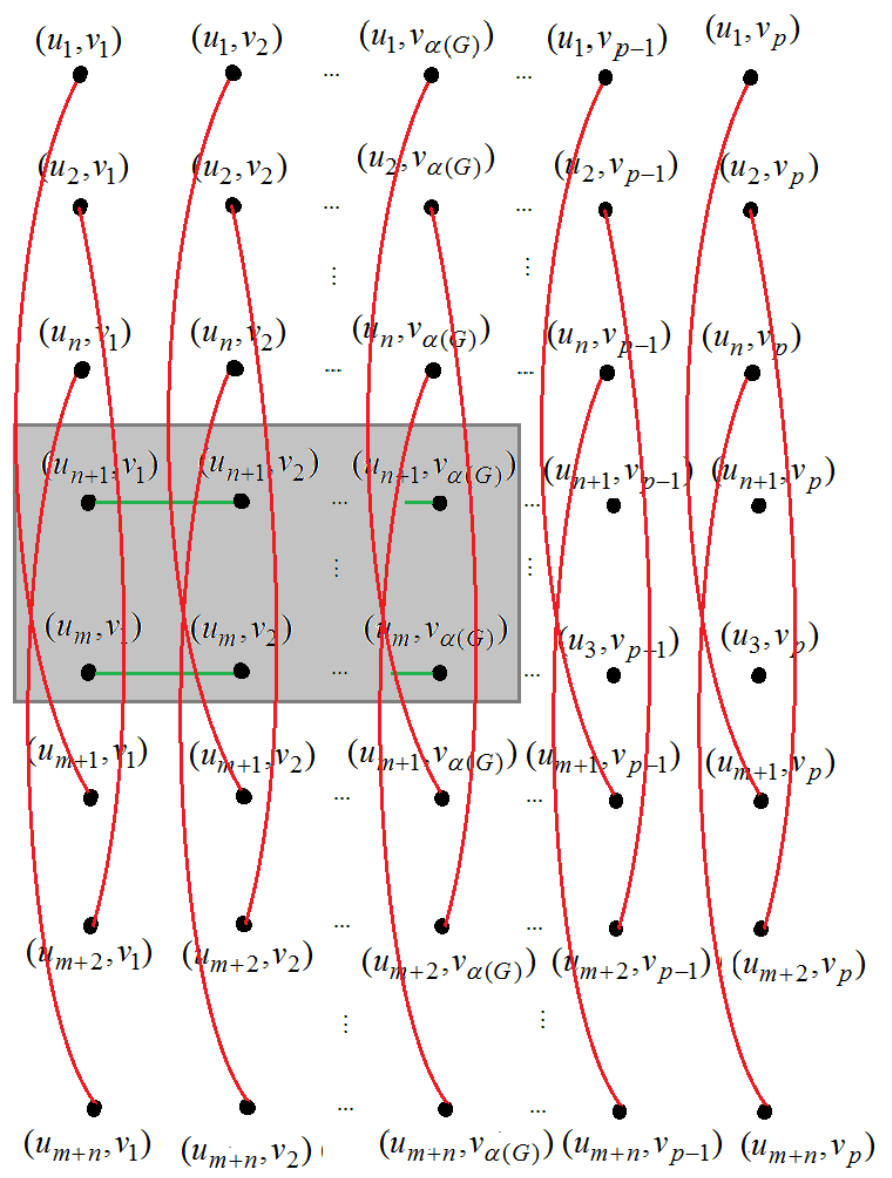

Figure 3: The case of $m>n$

Case 1. $m=n$.

$$
\begin{aligned}
\beta^{\prime}\left(K_{m, n} \otimes G\right) & =2 m p-p m \\
& =m p
\end{aligned}
$$

Hence $\beta^{\prime}\left(K_{m, n} \otimes G\right)=m p$, where $m=n$.

Case 2. $m>n$.

$$
\begin{aligned}
\beta^{\prime}\left(K_{m, n} \otimes G\right) & =(m+n) p-p n+(n-m) \alpha^{\prime}(G) \\
& =m p+(n-m)\left(p-\beta^{\prime}(G)\right)
\end{aligned}
$$




$$
\begin{aligned}
& =n\left(p-\beta^{\prime}(G)\right)+m \beta^{\prime}(G) \\
& =n p+(m-n) \beta^{\prime}(G)
\end{aligned}
$$

Hence $\beta^{\prime}\left(K_{m, n} \otimes G\right)=n p+(m-n) \beta^{\prime}(G)$, where $m>n$.

Case 3. $m<n$.

$$
\begin{aligned}
\beta^{\prime}\left(K_{m, n} \otimes G\right) & =(m+n) p-p m+(m-n) \alpha^{\prime}(G) \\
& =n p+(m-n)\left(p-\beta^{\prime}(G)\right) \\
& =m\left(p-\beta^{\prime}(G)\right)+n \beta^{\prime}(G) \\
& =m p+(n-m) \beta^{\prime}(G)
\end{aligned}
$$

Hence $\beta^{\prime}\left(K_{m, n} \otimes G\right)=m p+(n-m) \beta^{\prime}(G)$, where $m<n$.

\section{Acknowledgements}

This research was funded by King Mongkut's University of Technology North Bangkok. Contract no. KMUTNB-GEN-57-15.

\section{References}

[1] A. Vesel and J. Zerovnik, The independence number of the strong product of odd cycles, Discrete Math. 182,333-336, (1998).

[2] B.H. Barnes and K.E. Mackey, A generalized measure of independence and the strong product of graphs, Networks 8, 135-151, (1978).

[3] D.B.West, Introduction to Graph Theory, Prentice-Hall,(2001).

[4] P.K. Jha and G. Slutzki, Independence numbers of product graphs, Appl. Math. Left. 7 (4), 91-94, (1994).

[5] C.Baitiang, T.Sitthiwirattham, Independent and Vertex Covering number on strong product of complete graph, International Journal of Pure and Applied Mathematics. 81(3), 497-503, (2012).

[6] T.Sitthiwirattham, C.Baitiang, Matching and Edge Covering Number on strong product of complete graph, Far East Journal of Mathematical Science. $75(1), 165-172,(2013)$. 
[7] S. Chasreechai, T.Sitthiwirattham, Independent and Vertex Covering number on strong product of complete bipartite graph, Far East Journal of Mathematical Science. 83(1), 41-48, (2013). 
